\title{
Operational Damage Localization of Wind Turbine Blades
}

\section{Conference Paper}

Author(s):

Ou, Yaowen W.; Dertimanis, Vasilis K.; Chatzi, Eleni (D)

Publication date:

2017

Permanent link:

https://doi.org/10.3929/ethz-b-000228193

Rights / license:

In Copyright - Non-Commercial Use Permitted

Originally published in:

Lecture Notes in Civil Engineering 5, https://doi.org/10.1007/978-3-319-67443-8_22

\section{Funding acknowledgement:}

679843 - Smart Monitoring, Inspection and Life-Cycle Assessment of Wind Turbines (EC) 


\title{
Operational Damage Localization of Wind Turbine Blades
}

\author{
Yaowen W. Ou, Vasilis K. Dertimanis, and Eleni N. Chatzi ${ }^{(\bowtie)}$ \\ Department of Civil, Environment and Geomantic Engineering, \\ ETH Zurich, Zurich, Switzerland \\ chatzi@ibk. baug. ethz.ch
}

\begin{abstract}
This study tackles the challenge of operational damage identification on wind turbine blades and proposes a novel framework for damage detection and localization. A vibration-based scheme is proposed, which tracks the variability of the mode shape curvatures (MSCs) of the blade along its plane direction. The method consists of a training and a diagnostics stage. In the former, MSC information for a number of predefined modes is extracted over varying operational conditions in the healthy state of the blade and, via the implementation of the principal component analysis (PCA), a statistical characterization of each blade's node is estimated. Then, during the diagnostics stage, the MSCs are assembled and the same PCA mapping is enforced. A corresponding damage index is established, in order to detect and localize damage, if it exists. In both stages, MSC extraction is based on the successful estimation of vector autoregressive moving average (VARX) models that rely on pressure excitation and distributed strain measurements.
\end{abstract}

Keywords: Wind turbines - Operational conditions - Damage localization · Principal component analysis - Mode shape curvatures

\section{Introduction}

Structural damage detection and localization forms a scientifically difficult and technologically important issue that has, over the past years, attracted the attention of researchers, engineers, as well as infrastructure stakeholders and insurance companies. Among other candidate approaches, vibration-based structural health monitoring methods have been extensively investigated, as a result of their sound theoretical basis, and the further support provided through well-established and cost effective instrumentation schemes $[1,2]$.

Focusing on structures that operate on harsh and diverse conditions, such as wind turbine blades, an important aspect of this problem pertains to decision-support, for powering off or intervening for inspection/repair of these components, in light of the variability of the environments they are exposed to. A major challenge lies in the notable influence exerted on damage-sensitive features, such as natural frequencies and mode shapes, by the aforementioned environmental influences [3,4]. These effects are more extensively documented on bridge structures, with an approximately 5\% frequency variation on the Alamosa Canyon bridge reported in Farrar et al. [5] due to changing 
environmental conditions, while Alampalli [6] reports an up to 50\% variation in natural frequencies of an abandoned bridge in Claverack (NY) as a result of freezing of the supports.

Available methodologies for incorporating or discarding operational variability from structural models and associated indicators may be cast in two prominent categories [7]: (i) output-only methods, relying on response data alone, which seek to remove environmental influences from extracted vibrational features [8, 9], and (ii) input-output methods, which instead aim to determine a functional relationship between the response features and the measured operational conditions $[10,11]$. In the first class of methods, PCA [12] or its nonlinear ramifications (kernel PCA [13], Factor Analysis), holds a prominent role in solving the unsupervised learning problem by identifying patterns that are indicative of the effects of the unobserved inputs.

Powered by PCA on modal frequencies, Yan et al. $[14,15]$ detect damage in both experimental and simulated data under varying environments. Bellino et al. [16] propose a PCA approach suitable for time-varying systems, a topic of particular interest for wind turbine facilities. Nguyen and coworkers [17] suggest a temporal and spectral implementation of PCA for treatment of eigenfrequency and modal shape features respectively, extracted from a bridge in Luxemburg for the purpose of damage detection and localization.

Surprisingly, while the MSCs have been extensively used for damage detection and localization, they have not been exploited to such an end under the influence of changing operational and environmental conditions. For instance, Limongelli et al. [18] employ PCA and MSCs to localize artificial damage on a cantilever aluminum beam tested in the laboratory, under the premise however of invariant environmental conditions. In incorporating such a consideration, Shokrani et al. [19] recently introduced a framework for exploiting MSCs for damage localization, demonstrated on the numerical case study of a bridge system. The present study advances this approach for implementation with simulations of dynamic strain data, extracted from an operational wind turbine blade under temperature variations. For this purpose and under normal operating conditions, the variation of MSCs is tied to the independent sources of operational variability. Thus, during a training stage, the MSCs formulated across a representative operational period, a residual matrix is calculated and a statistical characterization of each node of the blade is derived. Accordingly, in every diagnostic stage, the corresponding MSCs are decomposed using the PCA information established at the training stage and an induced damage index is utilized for achieving damage detection and localization. It is noted that the method uses pressure excitation and distributed strain responses. The latter are deemed preferable when fatigue analysis is of interest, they do not take much space, while recent sensor technologies (e.g. Fiber Bragg Grating) indicate that strain sensors can be embedded into composite materials and integrated into several arrays, which imply structural information from a large number of points [20]. The method can be directly applied, however, to other types of excitation response pairs as well. 


\section{Blade Description}

\subsection{Finite Element Model}

Figure 1(a) displays the FE model geometry of the wind turbine blade considered, which is $1750 \mathrm{~mm}$ long and weighs approximate $5 \mathrm{~kg}$. It consists of woven materials by E-glass and Polyurethane resin matrix, as well as four shear webs in root region. The finite element (FE) model is constructed using shell elements for the outer surfaces of the blade with orthotropic material of equivalent mechanical properties (relying on the specifications provided by the manufacturer).

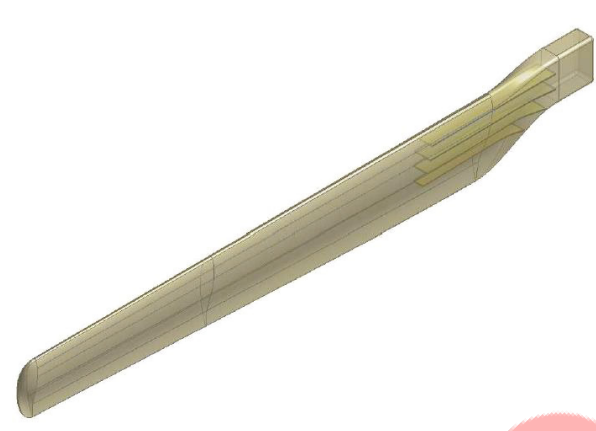

(a) ANSYS Workbench ${ }^{\circledR}$ FE model geometry

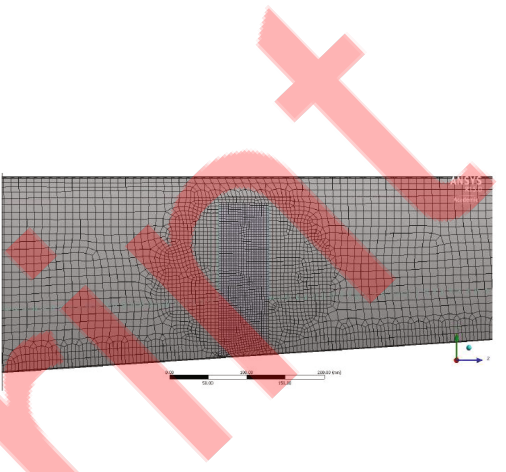

(b) ANSYS Workbench ${ }^{\circledR}$ FE model damage detail

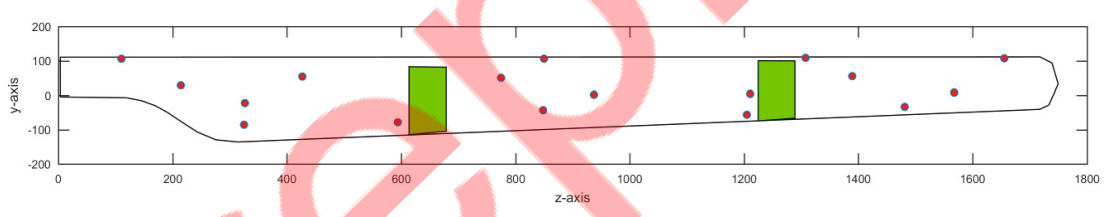

(c) Blade's plane view, showing the axes, the boundary, the damage areas and the sparse data configuration

Fig. 1. Details of the blade's numerical model.

Accurate simulation of the blade in its healthy and damaged states is ensured by careful meshing of the associated geometry, especially with respect to the contact regions among the different blade constitutive materials and elements. This applies specifically to the contact regions between (i) the shell surface and the core foam, (ii) the shear webs' edges and the outside shell surface, (iii) the shear webs' edges and the core foam, and (iv) the shear webs' faces and the core foam. A bonded contact type has been realized for these regions, implying no relative movement (with or without friction) among the edge-surface contacts during simulation.

Following the work described in $\mathrm{Ou}$ et al. [21], the FE model is subsequently updated using nonlinear optimization. This is accomplished by minimizing the relative error between the first six natural frequencies and those obtained through the experimental modal analysis data on the actual blade. The reference temperature of the updating process is $20^{\circ} \mathrm{C}$. 
In order to include a realistic operational framework, the working temperature of blade is arranged from $-15{ }^{\circ} \mathrm{C}$ to $40{ }^{\circ} \mathrm{C}$ and it is decomposed into 12 uniform intervals of 5 degrees. As temperature changes, the Young's modulus and the shear modulus in all directions are reduced in accordance to existing thermal constitutive equations [22-24]. The Poisson ratios remain constant, while the thermal energy conduction time is ignored, since the analysis is carried out in the steady-state.

\subsection{Damage Areas}

As Figs. 1(b) and (c) indicate, two typical damage locations at approximately 35\% (location A) and 70\% (location B) along the $\mathrm{z}$-axis in the flapwise direction of the blade are considered [25]. These are realized as a percentage reduction in the associated material properties within two areas of sizes [210 $\times 65] \mathrm{mm}$ and [185 $\times 65] \mathrm{mm}$. The mesh (obtained by applying shell elements) of the $35 \%$ damage area (closer to blade root) is illustrated in Fig. 1(b).

\subsection{Wind Pressure Excitation}

Blade excitation adopts the model described in $\mathrm{Ou}$ et al. [26], according to which the dynamic pressure applied is provided by

$$
P_{w}(t)=\frac{1}{2} C_{p} \rho u_{w}^{2}(t)
$$

where $C_{p}$ and $\rho$ denote the pressure coefficient (assumed constant) and the density of air, respectively, while $u_{w}$ is the wind speed load, given by the sum of an average speed $\left(u_{a}\right)$, a wind speed ramp $\left(u_{r}(t)\right)$, a wind gust $\left(u_{g}(t)\right)$ and a turbulence $\left(u_{t}(t)\right)$, as

$$
u_{w}(t)=u_{a}+u_{r}(t)+u_{g}(t)+u_{t}(t)
$$

The ramp and the gust components evolve over specific intervals, $\left[T_{s r}, T_{e r}\right]$ and $\left[T_{s g}, T_{e g}\right]$, respectively, and they are calculated by

$$
\begin{gathered}
u_{r}(t)=\left\{\begin{array}{cc}
A_{\text {ramp }} \frac{t-T_{s r}}{T_{e r}-T_{s r}}, & t \in\left[T_{s r}, T_{e r}\right] \\
0, & \text { otherwise }
\end{array}\right. \\
u_{g}(t)= \begin{cases}A_{\text {gust }}\left(1-2 \cos \left(2 \pi \frac{t-T_{s g}}{T_{e g}-T_{s g}}\right)\right), & t \in\left[T_{s g}, T_{e g}\right] \\
0, & \text { otherwise }\end{cases}
\end{gathered}
$$

with $A_{\text {ramp }}$ and $A_{\text {gust }}$ corresponding to the ramp and gust amplitudes, respectively. The turbulent component is given by

$$
u_{t}(t)=\sqrt{2} \sum_{k=1}^{N}\left[P_{D}\left(\omega_{k}\right) \Delta \omega\right]^{1 / 2} \cos \left(\omega_{k} t+\varphi_{k}\right)
$$


where $\omega_{k}$ are frequencies in $\mathrm{rad} / \mathrm{s}, \varphi_{k}$ are random phases in $[0,2 \pi]$ and $P_{D}\left(\omega_{k}\right)$ is a spectral density function calculated by

$$
P_{D}\left(\omega_{k}\right)=\operatorname{lu}\left(\ln \left(\frac{h}{z_{0}}\right)\right)^{-1}\left(1+1.5 \frac{\omega_{k} l}{u_{a}}\right)^{-\frac{5}{3}}
$$

In Eq. (6), $h(\mathrm{~m})$ is the height of the hub from the ground, $l(\mathrm{~m})$ is the turbulence length scale and $z_{0}(\mathrm{~m})$ is the roughness length. The spectral density values to be used in Eq. (5) are sampled by evenly discretizing $P_{D}\left(\omega_{k}\right)$ at $N_{\omega}$ frequency points, $\omega_{k}=k \Delta \omega$, for a resolution $\Delta \omega$ and a cutoff frequency $\omega_{c}=N_{k} \Delta \omega$.

\section{The Operational Damage Localization Framework}

The proposed method consists of two interrelated steps that take on (i) the acquisition of sufficient information describing the healthy operational regime of the blade, during a training stage; and (ii) the establishment of a robust hypothesis test, which provides information on where a damage has occurred, during a diagnostics stage. The method currently assumes the availability of pressure excitation and strain response data, but it can be easily adapted to any type of available vibration excitation-response pairs. Both stages are based on the effective identification of a representative number of blade's vibration modes and the subsequent estimation of a function that approximates the curvature of each vibration mode's shape. To this end, an estimation module is integrated to the method, which accepts vibration excitation-response data recorded at distinct operational points (either training, or diagnostics) and returns the curvature functions of the vibration modes.

\subsection{The Estimation Module}

During each operational point, a VARX model of the form

$$
\boldsymbol{\varepsilon}[t]+\sum_{k=1}^{n a} \mathbf{A}_{k} \boldsymbol{\varepsilon}[t-k]=\sum_{m=0}^{n b} \mathrm{~B}_{m} P_{w}[t-m]+\mathbf{e}[t]
$$

is fitted to the data, where $\varepsilon[t]$ is the $[s \times 1]$ vector of recorded strains at the monitored locations on the blade, $P_{w}[t]$ is the excitation pressure, $\mathbf{A}_{k}, \mathbf{B}_{m}$ are the $[s \times s]$ and $[s \times 1]$ coefficients of the associated AR and exogenous matrix polynomials, respectively, and $\mathbf{e}[t]$ is a zero mean vector Gaussian white noise with covariance matrix $\boldsymbol{\Sigma}_{e e}$.

VARX model estimation is quite standard and it reduces to a typical linear regression problem that is solved through ordinary linear least squares. The interested reader is referred to Ljung [27] for further details. Upon availability of a VARX model, the next step pertains to the derivation of its state-space realization [21, 28, 29]

$$
\begin{gathered}
\boldsymbol{\xi}[t+1]=\mathbf{F} \boldsymbol{\xi}[t]+\mathbf{G} P_{w}[t]+\mathbf{K e}[t] \\
\boldsymbol{\varepsilon}[t]=\mathbf{H} \boldsymbol{\xi}[t]+\mathbf{M} P_{w}[t]+\mathbf{e}[t]
\end{gathered}
$$


in which $\xi[t]$ is the $[s \cdot n a \times 1]$ state vector and $\mathbf{F}, \mathbf{G}, \mathbf{K}, \mathbf{H}, \mathbf{M}$ are the matrices of the state and the output equations, of appropriate orders. For the purposes of the current study, only $\mathbf{F}$ and $\mathbf{H}$ are of particular interest. The former is of size $[s \cdot n a \times s \cdot n a]$ and corresponds to the block companion matrix of the VAR matrix polynomial. By solving the eigenvalue problem of $\mathbf{F}$

$$
\mathbf{F} \Pi=\Pi \Lambda
$$

the eigenvectors $\boldsymbol{\Pi}$ and the eigenvalues $\boldsymbol{\Lambda}=\operatorname{diag}\left\{\lambda_{1}, \lambda_{2}, \ldots\right\}$ are extracted and the vibration modes of the blade are estimated [21]. Accordingly, "mode shape"-type information at the measurement locations may be retrieved by $\mathbf{\Phi}=\operatorname{real}\{\mathbf{H} \Pi\}$.

Based on the calculated values for $\boldsymbol{\Phi}$ and the boundary conditions of the blade, a "mode shape"-type function $f(y, z)$ is approximated using biharmonic spline interpolation [30] on a denser scattered data grid within the shape of the blade. Then, the MSCs along the two directions can be computed by

$$
\begin{aligned}
& \Psi_{j, y}(y, z) \equiv \frac{\partial^{2} f(y, z)}{\partial y^{2}}=\frac{f(y+\delta y, z)-2 f(y, z)+f(y-\delta y, z)}{\delta y^{2}} \\
& \Psi_{j, z}(y, z) \equiv \frac{\partial^{2} f(y, z)}{\partial z^{2}}=\frac{f(y, z+\delta z)-2 f(y, z)+f(y, z-\delta z)}{\delta z^{2}}
\end{aligned}
$$

with $j=1,2, \ldots, n$ denoting vibration mode.

\subsection{The Training Stage}

It is herein assumed that excitation-response data can be acquired from the blade in a distinct number of operational points $\tau_{1}, \tau_{2}, \ldots, \tau_{q}$, during the healthy state of the blade. At each such point, a VARX model is identified and the MSCs of Eqs. (10a), (10b) are extracted for $n$ vibration modes. By denoting all available grid nodes by $k=1,2, \ldots, p$ (a unique grid node corresponds to any $(z, y)$ point on the surface of the blade), a $[p \times q]$ matrix is defined for every mode and along each direction as

$$
\mathbf{D}=\left[\begin{array}{ccc}
\Psi\left[\tau_{1}, 1\right] & \cdots & \Psi\left[\tau_{q}, 1\right] \\
\vdots & \ddots & \vdots \\
\Psi\left[\tau_{1}, p\right] & \cdots & \Psi\left[\tau_{q}, p\right]
\end{array}\right]
$$

where the bracket notation implies MSC information in a specific operational point and at a specific node. Application of the PCA maps D by

$$
\mathbf{K}=\mathbf{T D}
$$

where $\mathbf{K} \in \mathbb{R}^{d \times q}$ and $\mathbf{T} \in \mathbb{R}^{d \times p}$ is an orthogonal matrix. Both $\mathbf{T}$ and $d$ are calculated by the singular value decomposition of the covariance matrix of $\mathbf{D}[14,19]$, while $d<<p$ corresponds to the number of all individual sources that affect the MSC under normal operating conditions and its choice is, in general, not critical. 
The loss of information from the mapping of Eq. (12) can be quantified by truncating the mapped data back to the original space

$$
\widehat{\mathbf{D}}=\mathbf{T}^{T} \mathbf{K}=\mathbf{T}^{T} \mathbf{T D}
$$

and by defining a residual matrix

$$
\mathbf{R}=\mathbf{D}-\widehat{\mathbf{D}}
$$

each row of which, say $[\mathbf{R}]_{l, *}$, evaluates the loss of information at a specific node. The aim of the training stage is thus to estimate the matrix $\mathbf{T}$, as well as to fit a statistical distribution to $[\mathbf{R}]_{l, *}$. For the current study it is assumed that the normal distribution is sufficient in characterizing the row samples of $\mathbf{R}$. It must be noted, however, that this assumption is not restrictive; it just determines the outlier detection method to be used during the diagnostics stage.

\subsection{The Diagnostics Stage}

Consider now the acquisition of excitation-response data at an arbitrary operational point $\tau_{\mathrm{I}}$. As before, the MSCs associated with this point are extracted via the estimation module, leading to a column vector $\mathbf{w}$ (for every mode and every direction considered) of the form

$$
\mathbf{w}=\left(\Psi\left[\tau_{\mathrm{I}}, 1\right], \Psi\left[\tau_{\mathrm{I}}, 2\right], \ldots, \Psi\left[\tau_{\mathrm{I}}, p\right]\right)^{T}
$$

To decide if the new data comes from a healthy or a damaged state of the blade, the vector $\mathbf{w}$ is appended to the matrix $\mathbf{D}$ to define

$$
\mathbf{D}_{\mathrm{I}}=[\mathbf{D w}]
$$

Just as in the training stage, projection and remapping of $\mathbf{D}_{\text {I }}$ implies

$$
\widehat{\mathbf{D}}_{\mathrm{I}}=\mathbf{T}^{T} \mathbf{T D}_{\mathrm{I}}
$$

and the updated residual matrix is

$$
\mathbf{R}_{\mathrm{I}}=\mathbf{D}_{\mathrm{I}}-\widehat{\mathbf{D}}_{\mathrm{I}}
$$

The latter residual matrix contains one additional sample in every row. Thus, a simple hypothesis test can be established, in order to determine if this new sample belongs to the estimated normal distribution of that row, implying that the new operational point comes from a healthy state of the blade, or if it does not, implying that this point likely comes from a damaged state of the blade. Under the assumption of normality, the adopted hypothesis test pertains to the classical Grubbs' test, where the statistic $G$ is compared against the critical value $G_{\mathrm{cr}}$ at a certain significance level $a$. 
If $G<G_{\text {cr }}$ the null Hypothesis (e.g. no damage) is adopted, otherwise the alternative Hypothesis (e.g. damage) is adopted instead. Based on this test, a damage index is defined in terms of the distance of the new sample from the mean value of the corresponding distribution

$$
\beta_{k}=\left\{\begin{array}{cl}
0, & G<G_{\mathrm{cr}} \\
\frac{\left|[\mathbf{R}]_{k, \tau_{\mathrm{I}}}\right|}{\sigma_{k}}, & G<G_{\mathrm{cr}}
\end{array}\right.
$$

for $\mathrm{k}=1,2, \ldots, p$. According to Eq. (19), a non-zero damage index not only implies the presence of the damage, but also indicates the exact position (or a set of positions), where this damage has occurred. It must be emphasized that there is no guarantee that under a specific damage the same index is "excited" in all monitored modes. Indeed a damage may be sensitive in only a subset of modes, or may not be captured at all.

\section{Results}

The proposed damage localization method is now applied and assessed via the small-scale wind turbine blade previously described. To this end, the blade is simulated within the selected temperature range in its healthy state, as well as in distinct damage states that pertain to both single and multiple damages. In every simulation, the excitation consists of the same mean wind $(12 \mathrm{~m} / \mathrm{s})$, ramp and gust components, and different realizations of the turbulent component. All numerical values for the associated quantities of the excitation are adopted from Ou et al. [26, Table 1].

Using the sparse data grid of Figs. 1(c), Figs. 2 and 3 display the performance of the damage index (Eq. (19)) when a 30\% damage in location $\mathrm{A}$ is induced, for the first six mode shapes of the blade and for MSCs calculated along both $z$ and $y$ axis. It is apparent that the damage index has been activated only in the first mode, maintaining almost negligible values in all other ones. Moreover, even if significant values are returned for the first mode of the $y$ axis (Fig. 3, top plot), these are located in very narrow areas for away from the one of the actual damage. Such narrow areas are also
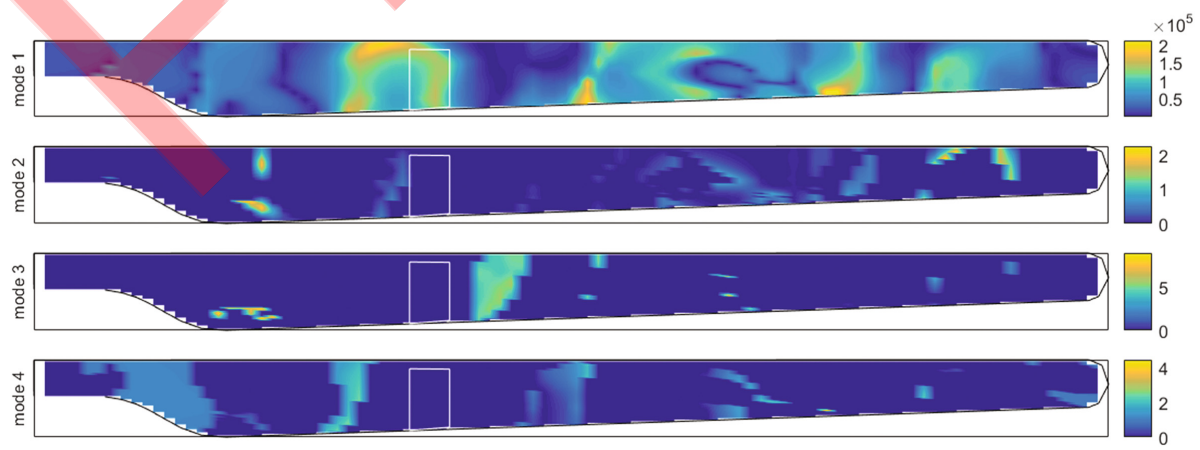

Fig. 2. Damage index for $30 \%$ stiffness reduction in location A (sparse grid, MSCs along $\mathrm{z}$ direction). 

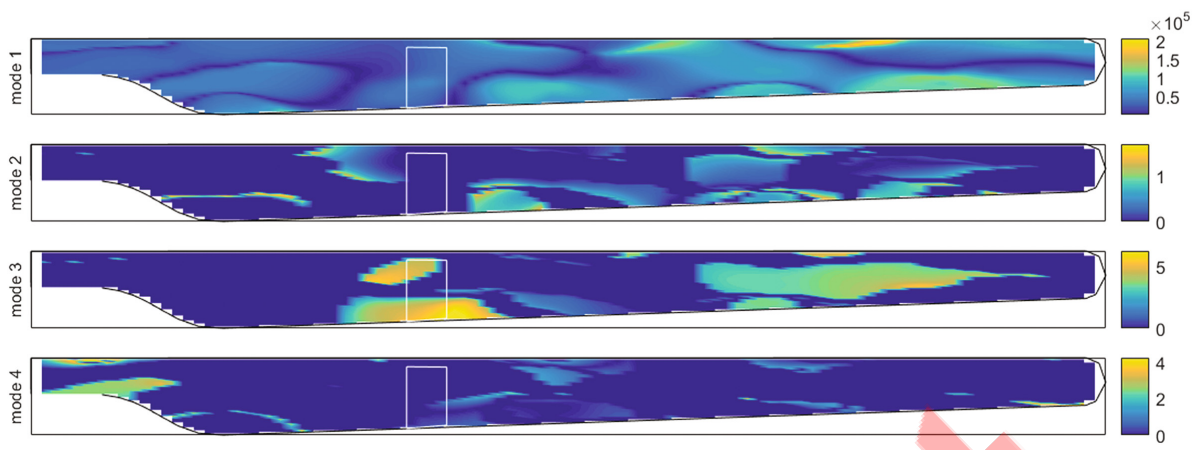

Fig. 3. Damage index for $30 \%$ stiffness reduction in location A (sparse grid, MSCs along y direction).

visible in the first mode of the $z$ axis (Fig. 2, top plot) and they deserve further attention, especially in respect to the underlying effects of the blade damage mechanisms. In any case, it is, however, obvious that the damage index has successfully detected and localized a significant area that lies exactly very close to the damage location A. Following these results, only the first mode shape's MSC along the $z$ axis is considered for further analysis and Fig. 4 illustrates the values of the damage index for $50 \%$ damage in location A. The damage has been successfully localized, while "false alarm" areas are sufficiently suppressed.

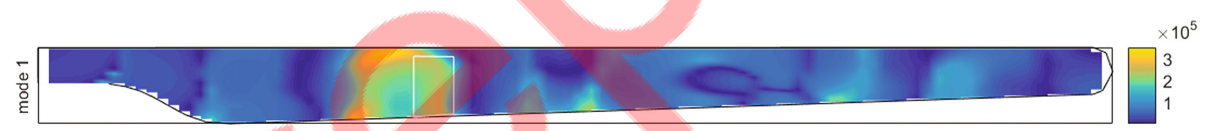

Fig. 4. Damage index for $50 \%$ stiffness reduction in location A (sparse grid, MSC along z direction).

The case of simultaneous damages at locations A and B is shown in Fig. 5, from which similar remarks can be made. Interestingly, the damage index is activated along the $z$ axis before the two locations, a feature that is rather attributed to the finite difference approximation of the MSCs. Notice also that difference damage levels may mask damages in other locations. This is apparent in Figs. 5(b)-(c), where the damage levels are different between locations and the index of the lower damage level is masked by the one of the higher damage level. An adaptive strategy for handling such cases, maybe through the use of structure-based information, is currently under investigation. 


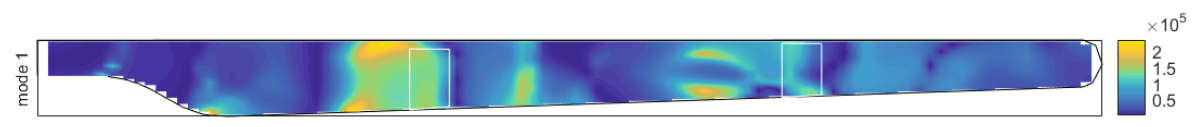

(a) $30 \%$ stiffness reduction in $A$ and both locations

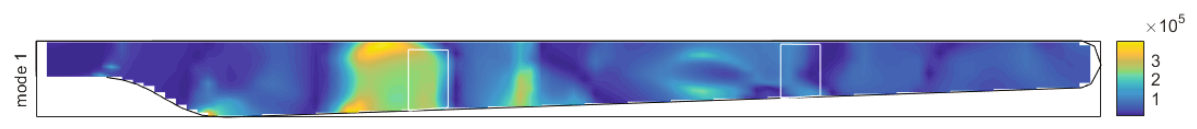

(b) $50 \%$ stiffness reduction in location $A$ and $30 \%$ stiffness reduction in location B

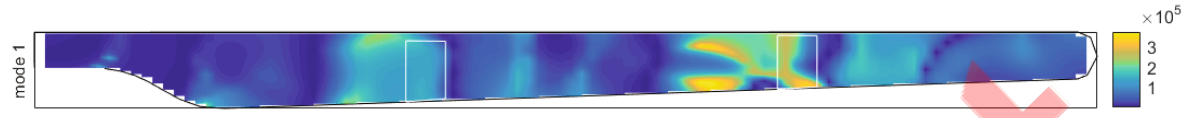

(c) $30 \%$ stiffness reduction in location $A$ and $50 \%$ stiffness reduction in location $B$

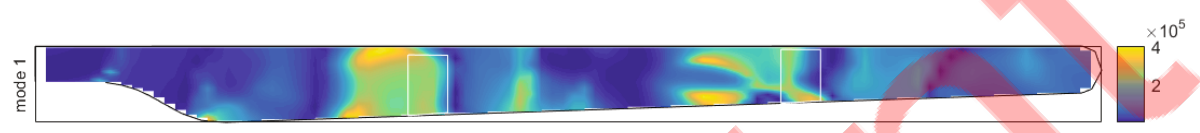

(d) $50 \%$ stiffness reduction in both locations

Fig. 5. Damage index for multiple damages along the blade (MSCs along z direction).

\section{Conclusions}

A novel vibration-based damage detection and localization method for wind turbine blades is outlined in this paper. The method accounts for environmental variability and explores the behavior of the blade's MSCs along distinct operational points. Based on an estimation module, during which a VARX model is estimated and the MSCs are extracted for a number of predefined modes, the proposed scheme constructs MSC data matrices and applies the PCA, in order to estimate all individual sources of variability. At a training level, this allows a statistical characterization of each individual node of the blade in its healthy state. Then, during a diagnostics stage, new data is appended to each node and the procedure is repeated using the quantities extracted at the training stage. A simple and effective hypothesis test and an associated damage index assigned to each node provide indications on whether damage has occurred, or if the new samples belong to the estimated distributions, implying that the blade is still undamaged.

As the numerical results indicate, the method seems capable in identifying and localizing both single and multiple damages at various levels. This encouraging performance suggests further consideration of the method as a potential candidate for damage localization under environmental variability. To this end, a number of important issues are currently under systematic investigation by the authors. These include the effects of data type and locations, the performance of the method in higher modes, its adaptation to output-only schemes (through the use, for example, of simple VAR models), the behavior of the underlying interpolation, as well as the validation of the method through laboratory and field data. 
Acknowledgments. Professor E. Chatzi would like to gratefully acknowledge the support of the ERC Starting Grant award ERC-2015-StG \#679843 on the topic of "Smart Monitoring, Inspection and Life-Cycle Assessment of Wind Turbines".

\section{References}

1. Farrar, C.R., Worden, K.: An introduction to structural health monitoring. Philos. Trans. R. Soc. Lond. A: Math. Phys. Eng. Sci. 365(1851), 303-315 (2007)

2. Carden, E.P., Fanning, P.: Vibration based condition monitoring: a review. Struct. Health Monit. 3(4), 355-377 (2004)

3. Cornwell, P., Farrar, C.R., Doebling, S.W., Sohn, H.: Environmental variability of modal properties. Exp. Tech. 23(6), 45-48 (1999)

4. Liu, C., De Wolf, J.T.: Effect of temperature on modal variability of a curved concrete bridge under ambient loads. J. Struct. Eng. 133(12), 1742-1751 (2007)

5. Farrar, C.R., Doebling, S.W., Cornwell, P.J., Straser, E.G.: Variability of modal parameters measured on the Alamosa Canyon Bridge. In: Proceedings of SPIE, Orlando, FL, USA, pp. 257-263 (1997)

6. Alampalli, S.: Effects of testing, analysis, damage, and environment on modal parameters. Mech. Syst. Signal Process. 14(1), 63-74 (2000)

7. Limongelli, M.P., Chatzi, E., Döhler, M., Lombaert, G.: Towards extraction of vibration-based damage indicators. Quantifying the Value of Structural Health Monitoring, COST Action TU1402 (2015)

8. Kullaa, J.: Elimination of environmental influences from damage-sensitive features in a structural health monitoring system. In: Proceedings of the 1st European Workshop on SHM, Paris, France, pp. 742-749 (2002)

9. Spiridonakos, M., Chatzi, E., Sudret, B.: Polynomial chaos expansion models for the monitoring of structures under operational variability. ASCE-ASME J. Risk Uncertain. Eng. Syst. Part A: Civ. Eng. 2(3), B4016003 (2016)

10. Yuen, K., Kuok, S.: Ambient interference in long-term monitoring of buildings. Eng. Struct. 32(8), 2379-2386 (2010)

11. Peeters, B., Maeck, J., De Roeck, G.: Vibration-based damage detection in civil engineering: excitation sources and temperature effects. Smart Mater. Struct. 10(3), 518-527 (2001)

12. Magalhães, F., Cunha, A., Caetano, E.: Vibration based structural health monitoring of an arch bridge: from automated OMA to damage detection. Mech. Syst. Signal Process. 28, 212-228 (2012)

13. Reynders, E., De Roeck, G.: Vibration-based damage identification: the Z24-bridge benchmark. In: Beer, M., Kougioumtzoglou, I.A., Patelli, E., Au, I.S.K. (eds.) Encyclopedia of Earthquake Engineering. Springer, Berlin (2015)

14. Yan, A.M., Kerschen, G., De Boer, P., Golinval, J.C.: Structural damage diagnosis under varying environmental conditions - part I: a linear analysis. Mech. Syst. Signal Process. 19(4), 847-864 (2005)

15. Yan, A.M., Kerschen, G., De Boer, P., Golinval, J.C.: Structural damage diagnosis under varying environmental conditions-part II: local PCA for non-linear cases. Mech. Syst. Signal Process. 19(4), 865-880 (2005)

16. Bellino, A., Fasana, A., Garibaldi, L., Marchesiello, S.: PCA-based detection of damage in time-varying systems. Mech. Syst. Signal Process. 24(7), 2250-2260 (2010) 
17. Ngyen, V.H., Mahowald, J., Maas, S., Golinval, J.C.: Use of time-and frequency-domain approaches for damage detection in civil engineering structures. Shock Vib. (2014). doi:10. $1155 / 2014 / 872492$

18. Limongelli, M.P.: An experimental evaluation of the reliability of a damage localization algorithm based on FRF interpolation. J. Civil Struct. Health Monit. 5(4), 427-439 (2015)

19. Shokrani, Y., Dertimanis, V., Chatzi, E., Savoia, M.: Structural damage localization under varying environmental conditions. In: 11th HSTAM International Congress on Mechanics, Athens, Greece (2016)

20. Dos Santos, F.L.M., Peeters, B., Van Der Vorst, R., Desmet, W., Góes, L.C.S.: The use of strain and mixed strain/acceleration measurements for modal analysis. In: 9th International Conference on Structural Dynamics, EURODYN 2014, Porto, Portugal (2014)

21. Ou, Y., Chatzi, E.N., Dertimanis, V.K., Spiridonakos, M.D.: Vibration-based experimental damage detection of a small-scale wind turbine blade. Struct. Health Monit. 16(1), 79-96 (2017)

22. Wang, Z., Zhou, Y., Mallick, P.K.: Effects of temperature and strain rate on the tensile behavior of shod fiber reinforced polyamide-6. Polym. Compos. 23(5), 858-871 (2002)

23. Guo, Z.S., Feng, J., Wang, H., Hu, H., Zhang, J.: A new temperature dependent modulus model of glass/epoxy composite at elevated temperatures. J. Compos. Mater. 47, 3303-3310 (2012)

24. Walsh, R.P., McColskey, J.D., Reed, R.P.: Low temperature properties of a unidirectionally reinforced epoxy fibreglass composite. Cryogenics 35(11), 723-725 (1995)

25. Sörensen, F.B., Jørgensen, E., Debel, P.C., et al.: Improved design of large wind turbine blade of fibre composites based on studies of scale effects (Phase 1)-summary report. Report no. Risø-R-1390(EN), Risø National Laboratory, Roskilde, Denmark (2004)

26. Ou, Y., Dertimanis, V.K., Chatzi, E.N.: Experimental damage detection of a wind turbine blade under varying operational conditions. In: Proceedings of USD2016, Leuven, Belgium (2016)

27. Ljung, L.: System Identification: Theory for the User. Prentice-Hall Inc., New Jersey (1999)

28. Lütkepohl, H.: New Introduction to Multiple Time Series Analysis. Springer, Berlin (2002)

29. Dertimanis, V.K., Koulocheris, D.V.: VAR based state-space structures: realization, statistics and spectral analysis. Lecture Notes in Electrical Engineering, vol. 85, pp. 301315. Springer, Heidelberg (2011)

30. Sandwell, D.T.: Biharmonic spline interpolation of GEOS-3 and SEASAT altimeter data. Geophys. Res. Lett. 14(2), 139-142 (1987) 